\title{
Upgrade for the NSTX Control Computer ${ }^{1}$
}

\author{
D. Mueller ${ }^{2}$, D. A. Gates ${ }^{2}$ and J.R. Ferron ${ }^{3}$ \\ ${ }^{2}$ Princeton Plasma Physics Laboratory, Princeton, NJ 08543-0451 \\ ${ }^{3}$ General Atomics, San Diego, CA 92186-9784
}

\begin{abstract}
The National Spherical Torus Experiment (NSTX) is a proof of scientific principle experiment as a magnetic fusion containment device. A primary goal of NSTX operations is control of the plasma current, position and shape in real time for a wide range of plasma pressure and current density profiles. In order to employ the best calculation of the plasma current, position and shape, it is planned to implement the equilibrium analysis code, EFIT[1], in real-time, RTEFIT[2]. EFIT inverts the Grad-Shafranov equation and performs a least squares fit to the magnetics data. RTEFIT is also capable of providing the plasma current profile and the plasma pressure profile from analysis of diagnostic data. The calculation time for RTEFIT using the present NSTX control computer system is comparable to the expected energy confinement time on NSTX and is thus slower than desired. A computer upgrade based upon 604e processors will permit the RTEFIT calculation loop to complete in about $3 \mathrm{~ms}$. The presence of the passive plates further complicates the control algorithm to be used in conjunction with RTEFIT. The planned approach is to measure the eddy currents in the passive platesand to use the transient response of the coils to minimize the total shell current effect.
\end{abstract}

\section{INTRODUCTION}

The Spherical Torus (ST) concept may provide an attractive path to a fusion reactor or volume neutron source. $[3,4]$. The NSTX will explore the physics of low aspect ratio in magnetically confined fusion plasmas in interesting regimes. Figure 1 shows a poloidal cross-section of NSTX with flux contours. The design of NSTX allows for plasma with major radius of $0.85 \mathrm{~m}$, minor radius of $0.68 \mathrm{~m}$, aspect ratio of 1.25 , plasma current (Ip) of $1 \mathrm{MA}$, toroidal magnetic field of $0.6 \mathrm{~T}$, plasma elongation $(\kappa)<2.2$ and triangularity $(\delta)<0.5$. Heating powers of up to $11 \mathrm{MW}$ (6MW of high harmonic fast waves (HHFW) and $5 \mathrm{MW}$ of $80 \mathrm{keV}$ deuterium neutral beam injection (NBI)) will be available. The ohmic heating solenoid is capable of providing about $1 \mathrm{~V}$-sec of inductive flux which is sufficient to initiate the discharge and ramp the plasma current up to $1 \mathrm{MA}$. Electron cyclotron resonance heating is available to facilitate breakdown and co-axial helicity injection (CHI)[5] will provide additional V-sec savings during start-up as well as the possibility of completely non-inductive current drive to about $500 \mathrm{kA}$. Tools to control the current profile include HHFW, CHI and NBI.

In order to explore the range of stability transport properties of the ST, it will be necessary to control many of

\footnotetext{
1 Work supported by USDOE Contract No. DE-AC02-76CH0307
}

the plasma variables. The most commonly controlled of these are Ip, $R, a, K$, and $\delta$ as well as the location of the X-points in diverted plasmas. These parameters have all been controlled in tokamaks by using a variety of approximations to provide information for feedback control. Modem computers now provide the opportunity to treat the analysis of discharge parameters more accurately and to use more fully the available diagnostic information. Full reconstruction of the discharge equilibrium can be provided by the fitting code EFIT[1]. In order to provide feedback in real time the code RTEFTT [2] was developed. RTEFIT reproduces the results of the full EFIT for the above mentioned parameters as well as the toroidal current density, pressure profile, internal inductance and beta in real time with sufficient accuracy and speed (with sufficient computer power) to allow feedback control on the desired parameters.

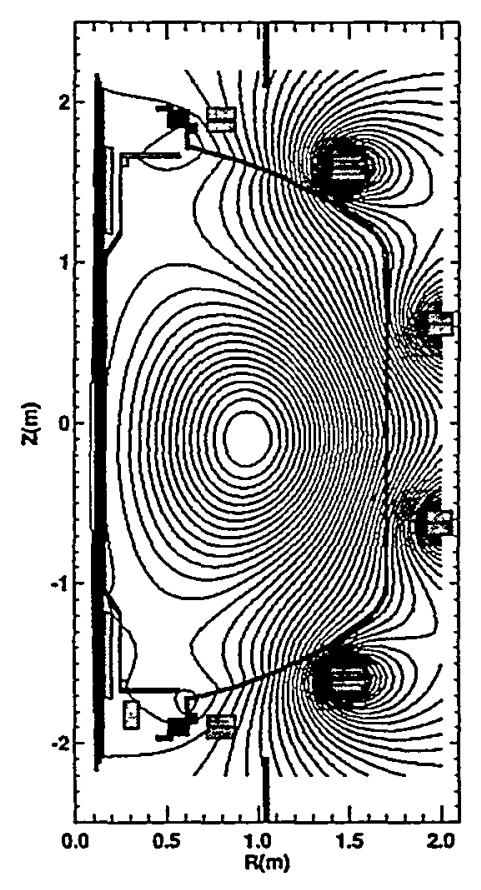

Figure 1. EFIT reconstruction of a 300kA NSTX plasma.

The present control computer for NSTX is a Skybolt I which employs 4 i860 processors for real-time control. This system is adequate for controlling the power supplies using analysis of the of plasma current, position and shape from the magnetics data. However, this system has nearly the same power as the DIII-D system that is based on 5 i860 processors. For DIII-D, the time to do a single iteration shape matrix $S$ 


\section{DISCLAIMER}

This report was prepared as an account of work sponsored by an agency of the United States Government. Neither the United States Government nor any agency thereof, nor any of their employees, make any warranty, express or implied, or assumes any legal liability or responsibility for the accuracy, completeness, or usefulness of any information, apparatus, product, or process disclosed, or represents that its use would not infringe privately owned rights. Reference herein to any specific commercial product, process, or service by trade name, trademark, manufacturer, or otherwise does not necessarily constitute or imply its endorsement, recommendation, or favoring by the United States Government or any agency thereof. The views and opinions of authors expressed herein do not necessarily state or reflect those of the United States Government or any agency thereof. 


\section{DISCLAIMER}

Portions of this document may be illegible in electronic image products. Images are produced from the best available original document. 
from which the plasma current distribution can be calculated is 22-30 ms, the loop to recalculate the plasma boundary shape given $S$ completes in 1-1.5 ms. Inclusion of other data to allow current and pressure profile information to be supplied would increase the slow loop to about $50 \mathrm{~ms}$. This is likely to be inadequate for the shorter confinement time scales expected on NSTX.

\section{SYSTEM REQUIREMENTS}

A long-term goal for NSTX is to control the plasma current and pressure profiles in order to improve performance. For this goal to be achieved, it will be necessary to provide real-time calculation of the plasma properties to be controlled. The most complete information will always be provided by off-line analysis. The RTEFIT code developed and used on DIII-D has been shown to be a robust way to provide rapid calculations that are a close approximation to the results obtained form off-line analysis (within $1 \mathrm{~mm}$ for the plasma boundary shape). The code allows for more complete diagnostic data to be included to provide information about the current profile in a straightforward way.

The requirements for real-time processor speed can be estimated from the experience with the DII-D RTEFIT analysis. The time to complete the loop to calculate the shape matrix, S, for DUI-D is from 22 to $30 \mathrm{~ms}$ using $31 \mathrm{~B}$-field measurements, 40 flux loops, Ip from a Rogowski loop, the measured coil currents and 5 to 7 fitting parameters for the current and pressure profiles. Initial RTEFIT analysis on NSTX will likely begin with a similar number of measurements, but will include more signals to properly deal with the effects of toroidal currents flowing in vacuum vessel and passive plates. Increasing the number of measurements will improve the accuracy, but will increase the calculation time.

When other diagnostics such as the Motional Stark Effect diagnostic (MSE) are used to diagnose the current density, the time to calculate $S$ will increase. For DIII-D, it is estimated that inclusion of this analysis will increase the loop time to $50 \mathrm{~ms}$. The goal of NSTX to use control of the plasma current profile to improve stability and confinement will eventually necessitate the inclusion of the MSE data. Current versions of the MSE diagnostic view a neutral beam injected into the plasma. The spectral splitting and polarization of emitted radiation provide a measure of the direction of the local magnetic field, from which the plasma current density can be inferred.[6] This technique is not useful without a neutral beam and the weak splitting in a low magnetic field coupled with the doppler broadened observed line width will limit its usefulness at low field. A technique using laser induced flourescence (LIF) to avoid the geometrical spectral broadening will allow MSE to be an effective diagnostic at low magnetic fields and will not require a neutral particle beam to be effective.[6]

The confinement scaling for an ST is not yet wellestablished, however, an energy confinement time in the range of $15-40 \mathrm{~ms}$ for NSTX is possible. In order to make the most useful calculation of the plasma properties, in is desirable to reduce the calculation time to less than the response of the power supplies. Practically, that means that providing real-time calculations based on diagnostic data in less than $2-3 \mathrm{~ms}$ is adequate.

In addition to equilibrium reconstruction and power supply control, the NSTX control computer will be used to provide other tasks. These tasks include control of the gas injection system (GIS), provision of a discharge fault system(DFS), operation of the CHI gas system and interfaces to the controls for the NBI and HHFW systems. The computational requirements for each of these tasks is small, however each does require real-time control with about lms response time.

Table 1 contains the number of input and output channels required for each of these systems. In each case the number of I/O channels will depend upon the details of the implementation and upon the amount of control logic that is handled by local controllers.

Table 1

I/O channels for various systems

\begin{tabular}{|l|l|l|}
\hline SYSTEM & Number of inputs & Number of outputs \\
\hline GIS & 4 to 20 & 6 to 14 \\
\hline CHI gas & 2 to 4 & 2 to 4 \\
\hline DFS & 3 & 3 \\
\hline NBI & 6 & 3 \\
\hline HHFW & 2 & 2 \\
\hline
\end{tabular}

\section{PRESENT CONTROL SYSTEM}

The control of the plasma current and position was accomplished by closed loop control of the current in the magnetic field coil power supplies during the first NSTX plasma operation in February 1999. The rectifiers are controlled digitally by a Skybolt I system comprised of a THEMIS host computer and 4 i860 real-time processors. This system will be used during the upcoming NSTX run period to control the plasma current and position with closed loop feedback.

The total toroidal current measurement is provided by a Rogowski coil that encloses the poloidal cross-section of the vacuum vessel. From this total toroidal current, the current flowing in the vacuum vessel and other toroidally continuous structures must be subtracted to provide the plasma current. These other toroidal currents are measured using either Rogowski coils which enclose the structural elements or by measurements of the loop voltage and knowledge of the structural resistance and are then subtracted in an analog circuit from the total toroidal current.

The present control system will acquire data from 7 flux loops, 3 B-field measurements, 44 coil and rectifier currents and the plasma current for use in determining the voltage requests for each of the 11 coil systems in the next $1 \mathrm{~ms}$ time step. It would be possible to perform an RTEFIT analysis with this system with a similar time response as that for the DII-D system. However, to provide better resolution of the plasma shape, more input channels will be required and hence more real time data acquisition capability will be required. 


\section{UPGRADE PATH}

The Skybolt II 6U VME Multiprocessor system with 4 $604 \mathrm{e}$ processors has approximately 8 times the compute speed of the present system. This system is planned to be implemented initially with 192 input channel capability at 100 $\mathrm{kHz}$ (this can be upgraded to 768 channels). The planned implementation will allow for 63 channels each of flux loop and B-field measurements, 2 plasma current measurements and 64 channels of coil current and power supply data. The results of offline EFIT analysis and RTEFIT simulations will be used to determine which and how many flux loops, B-field measurements and how many measurements of structural currents will be included in the final system. Under the assumption that the amount of data chosen will be similar to that for DIII-D, the new system will be able to calculate the $S$ matrix in 3 to $6 \mathrm{~ms}$ and the fast loop will be completed in less than $1 \mathrm{~ms}$. This speed should be adequate for NSTX control. In the near future, a daughter board with $4 \mathrm{G} 4$ processors will be available. If that happens before the system is purchased this fall, the higher speed processors (about a factor of 2) will be purchased instead of the 604e processors.

This system capability can be increased by the addition of a $6 \mathrm{U}$ motherboard and a daughterboard with either $4604 \mathrm{e}, 4$ i860, or 12 SHRAC processors. Such an addition will likely be made when the GIS, CHI gas and DFS are brought into the upgraded control system of NSTX. The decision about how completely to integrate these systems into the control computer will be made on the basis of cost-effectiveness and upon evaluation of the performance of the rudimentary systems in place for the initial NSTX operations.

\section{SUMMARY}

The upgrade control computer planned for NSTX is powerful enough to accommodate all planned data acquisition and has computing speed that is sufficient to permit state-ofthe-art plasma shape analysis to be completed in about the rectifier response time. This speed will introduce a delay in the feedback loop response of less than $1 \mathrm{~ms}$. The system computing speed and data acquisition can be further upgraded with minimal impact to the already installed portion of the system.

\section{ACKNOWLEDGEMENTS}

We wish to express our appreciation to the NSTX team and in particular to $\mathrm{F}$. Levinton who provided useful discussions on the MSE diagnostic and to C. Neumeyer, J. Menard, S. Kaye, T. Gibney and B. Penaflor who were essential to the early successes of the NSTX control system.

\section{REFERENCES}

1 L.L. Lao et al., Nucl. Fusion, 25, p. 1611 (1985).

2 J.R. Ferron et al., "Real Time Equilibrium Reconstruction for Tokamak Discharge Control", Nucl. Fusion, 38, pp. 1055-1066, July 1998.

3 M. Peng et al., Proc. $15^{\text {th }}$ Int. Conf. Plasma Physics and Controlled Nuclear Fusion Research, Seville, Spain, Plasma Phys. Cont. Nuclear Fusion Res., vol 2, p. 643 (1995).

4 R. Stambaugh et al., Proc. 16 $6^{\text {th }}$ Int. Conf. Plasma Physics and Controlled Nuclear Fusion Research, Montreal, Canada, Fusion Energy 1996, vol 2, p. 395 (1997).

5 T. Jarboe et al., Proc. 16 ${ }^{\text {th }}$ Int. Conf. Plasma Physics and Controlled Nuclear Fusion Research, Montreal, Canada, Fusion Energy 1996, vol 2, p. 243 (1997).

F.M. Levinton, "The motional Stark effect: Overview and future development (invited)", Rev.Sci. Instrum. vol.70, pp. 810-814, Jan. 1999. 